\title{
The Adaptation, Face, and Content Validation of a Needs Assessment Tool: Progressive Disease for People with Interstitial Lung Disease
}

\author{
Jason W. Boland, PhD, Carla Reigada, PhD, Janelle Yorke, $\mathrm{PhD}$, Simon P. Hart, $\mathrm{PhD},{ }^{1,3}$ \\ Sabrina Bajwah, $\mathrm{PhD}^{4,5}$ Joy Ross, $\mathrm{PhD}{ }^{6}$, Athol Wells, $\mathrm{MD}^{6}$, Athanasios Papadopoulos, $\mathrm{PhD}^{7}$ \\ David C. Currow, PhD, Gunn Grande, PhD, Una Macleod, PhD, and Miriam J. Johnson, MD ${ }^{1}$
}

\begin{abstract}
Background: Irrreversible interstitial lung disease (ILD) is associated with high morbidity and mortality. Palliative care needs of patients and caregivers are not routinely assessed; there is no tool to identify needs and triage support in clinical practice.

Objective: The study objective was to adapt and face/content validate a palliative needs assessment tool for people with ILD.

Methods: The Needs Assessment Tool: Progressive Disease-Cancer (NAT:PD-C) was adapted to reflect the palliative care needs identified from the ILD literature and patient/caregiver interviews. Face and content validity of the NAT:PD-ILD was tested using patient/caregiver focus groups and an expert consensus group. Participants in the study were two English tertiary health care trusts' outpatients clinics. There were four focus groups: two patient $(n=7 ; n=4)$; one caregiver $(n=3)$; and one clinician $(n=8)$. There was a single caregiver interview, and an expert consensus group-academics $(n=3)$, clinicians $(n=9)$, patients $(n=4)$, and caregivers $(n=2)$. Each item in the tool was revised as agreed by the groups. Expert consensus was reached. Results: Overall, the tool reflected participants' experience of ILD. Each domain was considered relevant. Adaptations were needed to represent the burden of ILD: respiratory symptoms (especially cough) and concerns about sexual activity were highlighted. All emphasized assessment of caregiver need as critical, and the role of caregivers in clinical consultations.

Conclusions: The NAT:PD-ILD appears to have face and content validity. The inclusion of the family caregiver in the consultation as someone with their own needs as well as a source of information was welcomed. Reliability testing and construct validation of the tool are ongoing.
\end{abstract}

\section{Introduction}

NTERSTITIAL LUNG DISEASE (ILD) represents a grouping of irreversible ILDs. Idiopathic pulmonary fibrosis (IPF) is the largest subgroup, with 5000 new cases annually in England and
Wales. ${ }^{1}$ IPF is a nonmalignant, progressive disease, ${ }^{2}$ with a median survival of three years. ${ }^{3,4}$ Common symptoms include breathlessness, cough, fatigue, and pain, often with psychosocial, financial, and spiritual distress. ${ }^{5}$ Despite national and international guidelines, ${ }^{6-9}$ assessments of palliative care

\footnotetext{
${ }^{1}$ Hull York Medical School, University of Hull, Yorkshire, United Kingdom.

${ }^{2}$ School of Nursing, Midwifery and Social Work, University of Manchester, Manchester, United Kingdom.

${ }^{3}$ Hull and East Yorkshire Hospitals NHS Trust, Hull, United Kingdom.

${ }^{4}$ King's College Hospital NHS Foundation Trust and, London, United Kingdom.

${ }^{5}$ Cicely Saunders Institute, London, United Kingdom.

${ }^{6}$ Royal and Harefield Trust Fundation, London, United Kingdom.

${ }^{7}$ School of Business, Management and Economics, University of Sussex, Brighton, United Kingdom.

${ }^{8}$ Palliative and Supportive Services, Flinders University, Adelaide, Australia.

Accepted January 13, 2016.

(C) Jason Boland, et al., 2016; Published by Mary Ann Liebert, Inc. This Open Access article is distributed under the terms of the Creative Commons Attribution Noncommercial License (http://creativecommons.org/licenses/by-nc/4.0/) which permits any noncommercial use, distribution, and reproduction in any medium, provided the original author(s) and the source are credited.
} 
needs are not part of routine clinical practice, and palliative services are rarely accessed. ${ }^{6,10}$ The National Institute for Health and Care Excellence (NICE) states that a needs assessment tool should be used for people with IPF. ${ }^{7}$ Several are available for people with cancer, ${ }^{11,12}$ but a clinician-rated tool to identify ILD patients with palliative care needs and triage referral to specialist palliative services does not currently exist.

The Australian Needs Assessment Tool:Progressive Disease-Cancer (NAT:PD-C) is a clinician-administered "aide-memoire" designed to help clinicians identify patients with palliative needs in daily practice and determine the added value of palliative care service involvement in the care of individual patients (for original questions, see Table 1, column 1). ${ }^{13-15}$ It has face and content validity, acceptability, and feasibility, ${ }^{13,14}$ and reduces unmet palliative care needs. ${ }^{15}$ However, relevance to ILD and the United Kingdom has not been assessed. The aim of this study was to adapt the NAT:PD-C for use in people with ILD (NAT:PD-ILD) and to test its face and content validity.

\section{Methods}

The NAT:PD-ILD adaptation was conducted in three steps (October 2014 to April 2015). Ethical approval was obtained prior to data collection from the National Research Ethics Service North East - Tyne \& Wear South (14/NE/0127).

\section{Step 1. Preliminary adaptation}

The NAT:PD-ILD was initially adapted from the NAT:PD-C by incorporating supportive and palliative care needs of patients with ILD and caregivers/relatives, identified from the literature and secondary analysis of qualitative patient/caregiver interviews. ${ }^{10,16-18}$

\section{Step 2. Focus group review}

The preliminary NAT:PD-ILD was reviewed by four focus groups (two patient groups; one carer group; one clinician group) and a single caregiver interview held on hospital premises.

\section{Participants}

Eligible patient participants were consenting adults attending ILD outpatients in two northern England sites. Current caregivers were invited through the patient or directly. Eligible clinicians were those caring for people with ILD in one health care region.

\section{Process}

The NAT:PD-ILD was further adapted after the clinician focus group. Following this, patient and caregiver focus groups and a single caregiver interview (using the same technique) were held. Participants were asked to discuss the face validity of the tool and review the adapted NAT:PD-ILD (including the instructions and prompts for clinicians) for content: relevance, appropriateness, whether the items reflected their personal experience. They were asked to suggest changes and/or to rectify omissions, which were incorporated in the NAT:PD-ILD.

\section{Step 3. Expert consensus group}

An expert consensus group was identified by the research team from their knowledge of their center teams and service user groups. The same process as for the focus groups was used. The final version of the NAT:PD-ILD was agreed on by the research team.

\section{Results \\ Participants}

The clinician focus group $(n=8)$ included three consultants (equivalent to attending physicians) and three specialist respiratory trainee physicians (between five and eight years postqualification), an ILD respiratory nurse specialist, and a physiotherapist. Further details are withheld to protect anonymity. Patients and caregiver characteristics are presented (see Box 1). The expert consensus group consisted of academics $(n=4)$, physicians $(n=5)$, nurses $(n=3)$, patients $(n=4)$, and caregivers $(n=2)$ from participating centers.

\section{Summary changes}

The changes made during the adaptation process from initial NAT:PD-C to the preliminary NAT:PD-ILD and then following focus group/interview and consensus group review to the final version are shown in Tables 1 and 2 , respectively.

\section{Findings and changes from the clinician focus group}

All agreed that the tool reflected their own practice. Most discussion focused on NAT:PD-ILD "red flags" identifying patients at risk of significant concerns. Additions related to ILD symptoms and support services were made. The caregiver sections were clearly important; caregivers' needs might be overlooked, particularly if they were absent from clinic. Clinicians were concerned about the balance of providing information and maintaining hope. The phrases suggested in the NAT:PD-ILD to help the clinician to introduce psychosocial-spiritual concerns were thought important to facilitate its use.

\section{Findings and changes from the patients and caregivers focus groups/interview}

Patients and caregivers considered that the NAT:PD-ILD was a holistic assessment guide, would aid difficult discussions, and would identify issues needing further attention easily missed by "busy people."

Patients focused on physical symptoms (especially cough-also bothersome for caregivers), the psychological burdens of ILD, and their effect on daily activities. Patients and caregivers described profound strain on relationships, especially marital, with changes of roles/function, including sexual issues. Patients described a loss of identity, feeling consumed by their illness, and feeling dependent on caregivers, which was isolating for both. Patients felt reluctant to "bother" clinicians by volunteering concerns and welcomed a tool that prompted clinicians to assess and legitimize issues.

The patient/caregiver experience was of "thinking about each other;" caregivers perceived that clinical consultations were focused solely on patient needs and excluded their contribution of important perspectives. They applauded the explicit assessment of their concerns/needs. Caregivers felt they took the psychological brunt of the patient's distress and frustrations, often felt lonely, and needed someone to talk to 
Centre for Health Research

and Psycho-oncology (2009)

NAT:PD-C version 0.0

Section 1: Priority referral for

further assessment

1. Does the patient have a caregiver readily available if required?

2. Has the patient or caregiver requested a referral to an SPCS?

3. Do you require assistance in managing the care of this patient and/or family?

Section 2: Patient well-being (Refer to the back page for assistance)

1. Is the patient experiencing unresolved physical symptoms (including problems with pain, sleeping, appetite, nausea, bowel, breathing, or fatigue)?

2. Does the patient have problems with daily living activities?

3. Does the patient have psychological symptoms that are interfering with well-being or relationships?

4. Does the patient have concerns about spiritual or existential issues?

5. Does the patient have financial or legal concerns that are causing distress or require assistance?

6. From the health delivery point of view, are there health beliefs, cultural or social factors involving the patient or family that are making care more complex?

7. Does the patient require information about (tick any options that are relevant): the prognosis; the cancer; treatment options; financial/legal issues; medical/health/support services; social/emotional issues

Section 3: Ability of caregiver or family to care for patient (Refer to the back page for assistance)

Who provided this information? (please tick one)

patient; caregiver; both

1 . Is the caregiver or family distressed about the patient's physical symptoms?

NAT:PD-ILD version 1.0

NAT:PD-ILD version 5.0

Section 1a: Red flags. If present, be alert for unmet palliative care need

Red flag symptoms

$\checkmark$ Clinical evidence of right heart failure

$\checkmark \mathrm{PO}_{2}<6 \mathrm{kPa}$ on air at rest and/or respiratory failure with raised $\mathrm{CO}_{2}$

$\checkmark$ Has the patient or carer had repeated unscheduled contact with the ILD team?

$\checkmark$ Failure to attend clinic today?

Section 1b: Priority referral for further assessment

$\checkmark$ No carer?

$\checkmark$ Patient or carer request referral to SPCS?

$\checkmark$ You require assistance of SPCS?

Section 2: Patient well-being ("Does

the patient have...")

Who provided this information?

patient; caregiver; both

$\checkmark$ Unresolved physical symptoms (including SOB, cough, leg edema, heartburn/ reflux/poor appetite, fatigue, insomnia/ daytime drowsiness, constipation, pain, cognition, self-image or sex)?

$\checkmark$ Unresolved psychological symptoms?

$\checkmark$ Problems with daily living activities?

$\checkmark$ Spiritual or existential concerns?

$\checkmark$ Financial or legal concerns?

$\checkmark$ Health beliefs, cultural or social factors making care delivery complex?

$\checkmark$ Information needs: prognosis; diagnosis; treatment options; financial/legal issues; support services; social/emotional issues

Section 3: Ability of carer or family to care for patient

("Is the carer /family...")

Who provided this information? (please tick one)

patient; caregiver; both

$\checkmark$ Distressed about the patient's symptoms?
Section 1a: Red flags. If present, be alert for unmet palliative care need

Red flag symptoms

$\checkmark$ Clinical evidence of right heart failure

$\checkmark$ Deteriorating performance status

$\checkmark$ Has the patient or carer had repeated unscheduled contact with hospitals?

$\checkmark$ Failure to attend clinic today?

Section 1b: Priority referral for further assessment

$\checkmark$ No carer?

$\checkmark$ Patient or carer request referral to SPCS?

$\checkmark$ You require assistance of SPCS?

Section 2: Patient well-being ("Does

the patient have...")

Who provided this information?

patient; caregiver; both

$\checkmark$ Unresolved physical symptoms (including SOB/ cough/ mucous; leg edema, heartburn/reflux/poor appetite, fatigue/insomnia/daytime drowsiness, constipation, pain, cognition, voice, sore mouth, mobility, self-image or sex)?

$\checkmark$ Unresolved psychological symptoms/loss of quality of life?

$\checkmark$ Problems with daily living activities?

$\checkmark$ Spiritual or existential concerns (issues about the meaning of life and suffering)

$\checkmark$ Work, financial, or legal concerns?

$\checkmark$ Health beliefs, cultural or social factors making care delivery complex?

$\checkmark$ Information needs: prognosis; diagnosis; treatment options; financial/legal issues; support services (social/emotional issues)

Section 3: Ability of carer or family to care for patient

("Is the carer/family...")

Who provided this information? (please tick one)

patient; caregiver; both

$\checkmark$ Distressed about the patient's symptoms? 
Centre for Health Research

and Psycho-oncology (2009)

NAT:PD-C version 0.0

2. Is the caregiver or family having difficulty providing

physical care?

3. Is the caregiver or family having difficulty coping?

4. Does the caregiver or family have financial or legal concerns that are causing distress or require assistance?

5. Is the family currently experiencing problems that are interfering with their functioning or interpersonal relationships, or is there a history of such problems?

6. Does the caregiver or family require information about (tick any options that are relevant): the prognosis; the cancer; treatment options; financial/legal issues; medical/health/support services; social/emotional issues

Section 4: Caregiver well-being (Refer to the back page for assistance)

Who provided this information? (please tick one)

patient; caregiver; both

1 . Is the caregiver or family experiencing physical, practical, spiritual, existential, or psychological problems that are interfering with their well-being or functioning?

2. Is the caregiver or family experiencing grief over the impending or recent death of the patient that is interfering with their well-being or functioning?

If referral required for further assessment or care, please complete this section

1. Referral to: (name)

2. Referral to: (specialty): general practitioner; social worker; psychologist; specialist palliative care service; medical oncologist; radiation oncologist; hematologist; other

3. Priority of assessment needed: urgent (within 24 hours); semi-urgent (2-7 days); non-urgent (next available)

4. Discussed the referral with the client: yes; no

5. Client consented to the referral: yes; no

6. Referral from: name: / position: / signature:

NAT:PD-ILD version 1.0

NAT:PD-ILD version 5.0

$\checkmark$ Having difficulty providing physical care?

$\checkmark$ Having difficulty coping?

$\checkmark$ Concerned about financial or legal issues?

$\checkmark$ Experiencing problems that are interfering with interpersonal relationships or functioning, or is there a history of such problems?

$\checkmark$ Information needs: prognosis, the diagnosis; treatment options; financial/legal issues; support services; social/emotional issues

Section 4: Carer/family well-being

("Carer or family

experiencing...")

$\checkmark$ Problems that are interfering with their well-being or functioning?

$\checkmark$ Grief over the impending death of the patient?
If further assessment required, please complete this section

$\checkmark$ Referral to: (name)

$\checkmark$ Specialty: ILD nurse specialist; pastoral care; psychology; OT; PT; social services; specialist palliative care; other

$\checkmark$ Priority of assessment needed: urgent (within 24 hours); semi-urgent (2-7 days); non-urgent (next available)

$\checkmark$ Patient aware of referral: yes; no

$\checkmark$ Patient agrees to referral: yes; no

$\checkmark$ Referral from: name: / position: / signature: $\checkmark$ Having difficulty providing physical care?

$\checkmark$ Having difficulty coping with the patient's psychological symptoms?

$\checkmark$ Concerned about financial or legal issues?

$\checkmark$ Experiencing problems that are interfering with interpersonal relationships or functioning, or is there a history of such problems?

$\checkmark$ Information needs: prognosis; the diagnosis; treatment options; financial/legal issues; support services (social) emotional issues)

Section 4: Carer/family well-being ("Carer or family experiencing...")

$\checkmark$ Unresolved psychosocial problems or feelings (loneliness, depression, anxiety, frustration) that are interfering with their well-being or functioning?

$\checkmark$ Grief over the future death of the patient?

If further assessment required, please complete this section

$\checkmark$ Liaison with:

$\checkmark$ Referral to: (name)

$\checkmark$ Specialty: ILD nurse specialist; spiritual care; psychology; OT; PT; social services; specialist palliative care; local/specialist ILD clinic; other

$\checkmark$ Priority of assessment needed: urgent (within 24 hours); semi-urgent (2-7 days); non-urgent (next available)

$\checkmark$ Patient aware of referral: yes; no

$\checkmark$ Copy to general practitioner: yes; no

$\checkmark$ Referral from: name: / position: / signature:

ILD, interstitial lung disease; $\mathrm{kPa}$, kiloPascals; NAT:PD-C, needs assessment tool: progressive disease-cancer; NAT:PD-ILD, needs assessment tool: progressive disease-interstital lung disease; OT, occupational therapy; PT, physical therapy; SOB, shortness of breath; SPCS, specialist palliative care service. 
Box 1. Demographics from Patient and Caregiver Focus Group/INTERVIEW ${ }^{\mathrm{a}}$

$\begin{array}{ll}\text { Mean age [range] } & \mathbf{7 0} \text { [55-89] years } \\ \text { Male } & 4 \\ \text { Mean time from diagnosis [range] } & 40[1-81] \text { months } \\ \text { Home-oxygen } & 9 \\ \text { Have a caregiver } & 8 \\ \text { White British } & 11 \\ \text { Caregivers focus group }(n=3) \text {; interview }(n=1) \\ \quad \text { Mean age [range] } & 64[64-65] \text { years } \\ \text { Male } & 1 \\ \text { White British } & 4\end{array}$

${ }^{\text {a Patients }} N=11$ (FG1=7; FG2=4).

$\mathrm{FG}$, focus group. for themselves. Caring consumed time and energy with consequent work and financial implications.

Patients and caregivers confirmed the need for opportunities to discuss the future and to address current major deficiencies in information, written or otherwise, about ILD and services available. They believed that palliative care was reserved for people dying with cancer and welcomed triaging access to specialist palliative care services. They expressed fear about dying, death, symptoms, and lack of help.

\section{Expert consensus group}

This group made minor changes to the NAT:PD-ILD. The final tool was agreed on by the study project management and steering group members.

TABle 2. Summary of NAT:PD-ILD Changes

4 sections (domains) and 1 referral section

\section{Section 1}

3 items concerning: if patient needs a carer; if patient or carer asks for PCS; if the clinician asks for help for this situation

Section 2 (about the patient)

7 items concerning: physical symptoms $(n=7)$; daily activities; psychological symptoms; spiritual or existential issues; financial or legal concerns; health beliefs, cultural or social factors; type of information needed

Section 3 (about carer \& patient)

6 items concerning: family

distressed and symptoms; physical care; coping; financial or legal concerns; interpersonal relationships; type of information needed

Section 4 (carer)

2 items concerning: caregiver problems (physical, practical, spiritual, existential, or psychological) and well-being; grief and well-being

\section{Referral section}

Specialties: general practitioner; social worker; psychologist; SPCS; medical oncologist; radiation oncologist; hematologist; other
4 sections (domains) and 1 referral section

Section 1 (2 subsections were created):

- 4 items concerning "red flags:" heart failure; $\mathrm{PO}_{2} / \mathrm{CO}_{2}$; ILD team contact; attend clinic

- 3 items concerning "priority

referral" (same as V0.0)

Section 2 (about the patient)

- Option of who provided

information was added

- Same 7 items, however:

More physical symptoms $(n=14)$ were added;

shorter sentences with the same content;

term "support services" to state "medical/health/support"

Section 3 (about carer \& patient)

- Same 6 items, however:

Shorter sentences with the same content;

term "the cancer" changed to

"the diagnosis," and

"medical/health/support" to "support services"

Section 4 (carer)

- Same 2 items, however:

Substantial changes in sentence structure

Referral section

- New specialties were added: ILD nurse specialist; pastoral care;

OT; PT

- Options as general practitioner; medical oncologist; radiation oncologist; hematologist were taken

- "Social worker" and

"psychologist" were changed

to "social services" and

"psychology"
4 sections (domains) and 1 referral section

Section 1

- $\mathrm{PO}_{2} / \mathrm{CO}_{2}$ issues changed to

"deteriorating performance status"

- "Unscheduled contact with hospitals" instead of "ILD team contact"

Section 2 (about the patient)

- Same 7 items, however:

More physical symptoms $(n=18)$ were added;

"loss of quality of life" was added/related to "unresolved psychological symptoms";

Explanation about spiritual dimension was added;

"work" added as a concern;

"support services (social/ emotional issues)" replace

"support services; social/ emotional issues"

- simplified

Section 3 (about carer \& patient)

- Same 6 items, however:

"Patient's psychological symptoms" was related to " coping",

"support services (social/ emotional issues)" replace

"support services; social/ emotional issues"

Section 4 (carer)

- Same 2 items, however:

Substantial changes in sentence structure

Referral section

- Local/specialist ILD clinic and "copy to general practitioner" were added

- "pastoral care", was changed to "spiritual care"

The initial four sections from NAT:PD-C were kept in the final NAT:PD-ILD tool. Two subsections were added in section 1. In section 2, specific ILD symptoms were added. In section 3, psychosocial variables were grouped. Section 4 had sentence structure changes. Other specialties to refer to were added in the referral section.

ILD, interstitial lung disease; NAT:PD-ILD, needs assessment tool: progressive disease-interstital lung disease; PCS, palliative care service; SPCS, specialist palliative care service. 


\section{Discussion}

The appearance and content of the adapted NAT:PD-ILD reflected the experience of patients, caregivers, and clinicians. Changes from the cancer-specific version were required to deal with differences between diseases and between service configuration.

The emphasis on respiratory symptoms by patients was not surprising, but cough was especially disruptive to caregivers. There was strong support for a caregiver assessment in the context of patient concerns. Caregivers felt marginalized, risking missed useful information about the patient and a lost opportunity for support. This echoes previous studies ${ }^{19}$ and contrasts with cancer services where there is public and professional awareness of the wide-reaching effects of cancer. ${ }^{20}$ Coordinated, multiprofessional services support the patient and family from prediagnosis through treatment to survivorship and end-of-life care, with evidence-based integrated palliative services available in many service models from diagnosis ${ }^{21}$ alongside a plethora of printed and online information and financial advice. ${ }^{22,23}$ In many countries, specialist palliative care services are less or not at all accessible to patients with nonmalignant disease. ${ }^{24,25}$

The model of multiprofessional holistic care in IPF is recommended by $\mathrm{NICE}^{26}$ but is poorly implemented, especially with regard to psychosocial support, advance care planning, and access to palliative care. Advanced communication skills training for cancer professionals was embedded in the National Cancer Plan 2000 in the United Kingdom $^{27}$ but is unavailable to ILD clinicians, despite the patient and caregiver distress associated with ILD.

In England and Wales, over 1.4 million people provide 50 hours or more unpaid care weekly. ${ }^{28}$ The impact of providing care with advancing disease and at the end of life for family members has been highlighted, ${ }^{29}$ but effective interventions to support the caregivers are less well defined. The NAT:PD-ILD could provide a valuable first step to identify and address caregiver needs alongside those of the patients.

\section{Implications for research}

Reliability testing and construct validation are ongoing. The cost effectiveness of the NAT:PD-ILD in reducing unmet need should be tested in well-designed clinical trials, including consideration of factors likely to affect implementation in practice, e.g., consultation time, clinician training needs, and resource implications. Consultation time did not increase with use of the original tool. ${ }^{30}$

\section{Implications for clinical practice}

Clinician-administered tools may prompt discussion of issues between patients, families, and health professionals, ${ }^{31,32}$ provide needs-driven management; ${ }^{33-35}$ help to prioritize resources; and identify areas for improvement. ${ }^{32,35}$ Although further work is needed, there are immediate clinical implications. First, caregivers should be encouraged to attend and contribute to the consultation, which should include an assessment of their needs. Second, ILD clinicians should access advanced communication and basic palliative care skills training. Last, there is an urgent need for education of the public and professionals that palliative care should be driven by individual needs and not restricted by diagnosis or perceived short-term prognosis.

\section{Strengths and limitations}

The use of multiple approaches drawing on clinical services from several areas in England is a strength. The focus groups were small and not widely representative. However, the findings are consistent with published literature and generated a tool that was endorsed by expert consensus.

\section{Conclusions}

The adapted NAT:PD-ILD appears to have face/content validity. Immediate implications for clinical practice include the need for active assessment of caregiver needs and palliative care and communication skills training for ILD clinicians. Reliability testing and construct validation are ongoing. Further study to test the cost effectiveness and practical implementation of the tool is required.

\section{Acknowledgments}

This study was funded by the Marie Curie Research Programme Project Award (C30598/A16976). We would like to thank the patients, carergivers, and clinicians who took part in this study for their time and insights.

\section{References}

1. Navaratnam V, Fleming KM, West J, et al.: The rising incidence of idiopathic pulmonary fibrosis in the U.K. Thorax 2011;66:462-467.

2. Collard HR, Anstrom KJ, Schwarz MI, Zisman DA: Sildenafil improves walk distance in idiopathic pulmonary fibrosis. Chest 2007;131:897-899.

3. Hubbard R, Johnston I, Britton J: Survival in patients with cryptogenic fibrosing alveolitis: A population-based cohort study. Chest 1998;113:396-400.

4. Hubbard R, Venn A: The impact of coexisting connective tissue disease on survival in patients with fibrosing alveolitis. Rheumatology (Oxford) 2002;41:676-679.

5. Swigris JJ, Stewart AL, Gould MK, Wilson SR: Patients' perspectives on how idiopathic pulmonary fibrosis affects the quality of their lives. Health Qual Life Outcomes 2005; 3:61.

6. Bradley B, Branley HM, Egan JJ, et al.: Interstitial lung disease guideline: The British Thoracic Society in collaboration with the Thoracic Society of Australia and New Zealand and the Irish Thoracic Society. Thorax 2008;63: v1-v58.

7. National Institute for Health and Care Excellence: Idiopathic pulmonary fibrosis. Quality statement 5: Palliative care. National Institute for Health and Care Excellence, 2015. www.nice.org.uk/guidance/qs79/chapter/Qualitystatement-5-Palliative-care (last accessed November 8, 2015).

8. Raghu G, Rochwerg B, Zhang Y, et al.: An official ATS/ ERS/JRS/ALAT clinical practice guideline: Treatment of idiopathic pulmonary fibrosis. An update of the 2011 clinical practice guideline. Am J Respir Crit Care Med 2015; 192:e3-e19.

9. Raghu G, Collard HR, Egan JJ, et al.: An official ATS/ ERS/JRS/ALAT statement: Idiopathic pulmonary fibrosis: Evidence-based guidelines for diagnosis and management. Am J Respir Crit Care Med 2011;183:788-824.

10. Bajwah S, Higginson IJ, Ross JR, et al.: Specialist palliative care is more than drugs: A retrospective study of ILD patients. Lung 2012;190:215-220. 
11. Carlson LE, Waller A, Mitchell AJ: Screening for distress and unmet needs in patients with cancer: Review and recommendations. J Clin Oncol 2012;30:1160-1177.

12. Richardson A, Medina J, Brown V, Sitzia J: Patients' needs assessment in cancer care: A review of assessment tools. Support Care Cancer 2007; 15:1125-1144.

13. Waller A, Girgis A, Currow D, et al.: Development of the palliative care needs assessment tool (PC-NAT) for use by multi-disciplinary health professionals. Palliat Med 2008;22: 956-964.

14. Waller A, Girgis A, Lecathelinais C, et al.: Validity, reliability and clinical feasibility of a needs assessment tool for people with progressive cancer. Psychooncology 2010;19: 726-733.

15. Waller A, Girgis A, Johnson C, et al.: Implications of a needs assessment intervention for people with progressive cancer: Impact on clinical assessment, response and service utilisation. Psychooncology 2012;21:550-557.

16. Bajwah S, Higginson IJ, Ross JR, et al.: The palliative care needs for fibrotic interstitial lung disease: A qualitative study of patients, informal caregivers and health professionals. Palliat Med 2013;27:869-876.

17. Bajwah KJ, Higginson IJ, Ross JR, et al.: "I wish I knew more...." The end-of-life planning and information needs for end-stage fibrotic interstitial lung disease: Views of patients, carers and health professionals. BMJ Support Palliat Care 2012.

18. Bajwah RJ, Peacock JL, Higginson IJ, et al.: Interventions to improve symptoms and quality of life of patients with fibrotic ILD: A systematic review of the literature. Thorax 2013, 68:867-879.

19. Bajwah S, Ross JR, Wells AU, et al.: Palliative care for patients with advanced fibrotic lung disease: A randomised controlled phase II and feasibility trial of a community case conference intervention. Thorax 2015;70:830-839.

20. Goodrich J, Cornwell J: Seeing the person in the patient: The point of care review paper. 2008. www.kingsfund.org.uk/ sites/files/kf/Seeing-the-person-in-the-patient-The-Pointof-Care-review-paper-Goodrich-Cornwell-Kings-FundDecember-2008.pdf (last accessed July 30, 2015).

21. National Institute for Health and Clinical Excellence: Improving Supportive and Palliative Care for Adults with Cancer. National Institute for Health and Clinical Excellence, 2004. www.nice.org.uk/guidance/csgsp/resources/ supportive-and-palliative-care-the-manual-2]. (Last accessed July 26, 2015.)

22. Advice and guidance about all the practical issues you might need to consider, including work, travel and finances. 2015. www.macmillan.org.uk/information-and-support/organising (last accessed July 25, 2015).

23. Cancer Research UK: Reliable, easy to understand patient information from Cancer Research UK. Cancer Research UK, 2015. www.cancerresearchuk.org/about-cancer/]. (Last accessed July 25, 2015.)
24. Boland J, Martin J, Wells AU, Ross JR: Palliative care for people with non-malignant lung disease: Summary of current evidence and future direction. Palliat Med 2013;27: 811-816.

25. Boland J, Johnson M: End-of-life care for non-cancer patients. BMJ Support Palliat Care 2013;3:2-3.

26. National Institute for Health and Clinical Excellence: Diagnosis and Management of Suspected Idiopathic Pulmonary Fibrosis: Idiopathic Pulmonary Fibrosis. National Institute for Health and Clinical Excellence 2013. www.nice.org.uk/ guidance/cg163]. (Last accessed July 26, 2015.)

27. Department of Health: The NHS Cancer plan: A plan for investment, a plan for reform. Department of Health, 2000. [webarchive.nationalarchives.gov.uk/20130107105354/http:// www.dh.gov.uk/prod_consum_dh/groups/dh_digitalassets/ @ dh/@en/documents/digitalasset/dh_4014513.pdf]. (Last accessed July 27, 2015.)

28. White C: 2011 Census Analysis: Unpaid care in England and Wales, 2011 and comparison with 2001. 2013. www .ons.gov.uk/ons/dcp171766_300039.pdf (last accessed August 2, 2015).

29. Round J, Jones L, Morris S: Estimating the cost of caring for people with cancer at the end of life: A modelling study. Palliat Med 2015;29:899-907.

30. Waller A, Girgis A, Johnson C, et al.: Improving outcomes for people with progressive cancer: Interrupted time series trial of a needs assessment intervention. J Pain Symptom Manage 2012;43:569-581.

31. Tamburini M, Gangeri L, Brunelli C, et al.: Assessment of hospitalised cancer patients' needs by the Needs Evaluation Questionnaire. Ann Oncol 2000;11:31-37.

32. Wen KY, Gustafson DH: Needs assessment for cancer patients and their families. Health Qual Life Outcomes 2004;2:11.

33. Davidson P, Cockburn J, Daly J, Sanson-Fisher R: Patientcentered needs assessment: Rationale for a psychometric measure for assessing needs in heart failure. J Cardiovasc Nurs 2004;19:164-171.

34. Deeken JF, Taylor KL, Mangan P, et al.: Care for the caregivers: A review of self-report instruments developed to measure the burden, needs, and quality of life of informal caregivers. J Pain Symptom Manage 2003;26:922-953.

35. Sanson-Fisher R, Girgis A, Boyes A, et al.: The unmet supportive care needs of patients with cancer: Supportive Care Review Group. Cancer 2000;88:226-237.

Address correspondence to: Jason W. Boland, PhD Hertford Building

Hull York Medical School University of Hull

Yorkshire, United Kingdom HU6 7RX

E-mail: jason.boland@hyms.ac.uk 